\title{
Perception of artificial stereoscopic stimuli from an incorrect viewing point
}

\author{
YOELLA BEREBY-MEYER, DAVID LEISER, and JOACHIM MEYER \\ Ben Gurion University of the Negev, Beer Sheva, Israel
}

\begin{abstract}
The present study investigates the distortions in the perception of artificial stereoscopic displays seen from an inappropriate distance and/or orientation. Stereoscopic displays represent 3-D information correctly, provided they are seen from the correct station point. The viewing point may differ from the correct station point in its distance or in its orientation to the screen. These differences lead to distortions that can be predicted mathematically. However, the perceptual function may be different from the predictions, since people may possibly compensate for the distortions. To test the degree of this compensation, participants saw anaglyphic stereoscopic stimuli that showed angles in the horizontal plane, and their perception of the configuration was tested for various orientations and distances. The estimates were compared with the values predicted from the mathematical functions, and participants' virtual positions were reconstructed via nonlinear regressions. The analyses revealed a moderate compensation for viewing orientations and a systematically overestimation of the viewing distances. These results indicate that people compensate partially for distortions in stereopsis, given that the relevant information is available.
\end{abstract}

Stereoscopic information enables an observer to perceive depth from the disparity of the two retinal images, resulting from the horizontal separation between the eyes. The geometrical relation between disparity and depth is (using the small angle approximation):

$$
\delta=\frac{I d}{D(D+d)},
$$

where $\delta$ is the angular binocular disparity, $D$ is the viewing distance defined as the distance to the nearer point, $d$ is the depth separation between two points, and $I$ is the interocular distance.

The retinal disparity that corresponds to a depth interval is approximately inversely proportional to the square of the viewing distance, when $d<<D$. In order to achieve veridical depth perception from disparity, the visual system must recalibrate disparity information for different viewing distances. The issue is known as the problem of stereoscopic depth constancy (Cormack, 1984; Fox, Cormack, \& Norman, 1987; Ono \& Comerford, 1977; Ritter, 1977, 1979).

The disparities that are obtained when real objects are viewed can be mimicked by presenting a pair of $2-D$ visual

The helpful comments of James Todd, Joel Norman, and Itshak Dinstein on earlier versions of this paper are gratefully acknowledged. This research was conducted under a grant from the Israeli Defense Ministry, Research and Development Branch. Thanks to Raphael Shefer, who programmed the experiments, and to Nava Ben-Artzy and Shely Drori, who helped to collect the data. Correspondence should be addressed to Y. Bereby-Meyer, Department of Education, or David Leiser, Department of Behavioral Sciences, Ben-Gurion University, Beer Sheva 84105, Israel (e-mail: yoella@bgumail.bgu.ac.il).

- Accepted by previous editor, Myron L. Braunstein displays separately to the eyes. The disparities and resulting depth perception are produced by the angular distance $(\delta)$ between corresponding points in the 2-D displays (Figure 1). In order to present a specific depth interval to an observer, the horizontal offset that corresponds to the disparity has to be computed for the specific viewing position, defined by the distance from the screen and orientation to the screen. The mathematical principles for the computation of disparity in artificial stereoscopic displays are given in the Appendix and have been elaborated elsewhere (Leiser, Bereby, \& Melkman, 1995). Any change in viewing position yields different magnitudes of depth. For instance, an anaglyphic-presented cube will flatten or expand as one moves nearer or farther from the screen, showing the distance effect, and will be distorted as one moves sideways, revealing the orientation effect.

The distance parameter has been studied, especially as related to the issue of stereoscopic depth constancy (Johnston, 1991; Patterson, Moe, \& Hewitt, 1992; Tittle, Todd, Perotti, \& Norman, 1995; Todd, Tittle, \& Norman, 1995; Wallach, Gillam, \& Cardillo, 1979), but the orientation effect has received little attention. The present study attempts to determine the effects of orientation on stereoscopic perception. Specifically, we sought to identify the nature of distortions that occur when artificial stereoscopic stimuli are seen from different orientations. The geometry of stereopsis allows quantitative predictions of the depth that should be perceived by the observer. For instance, changing the orientation of the viewer to the screen by an angle $\alpha$ will decrease the disparity from $\delta_{1}$ to $\delta_{2}$ by a factor of $\cos (\alpha)$, and the apparent depth of the virtual object should change accordingly (Figure 1).

However, perception is not necessarily determined by the geometry only. Compensational processes may inter- 


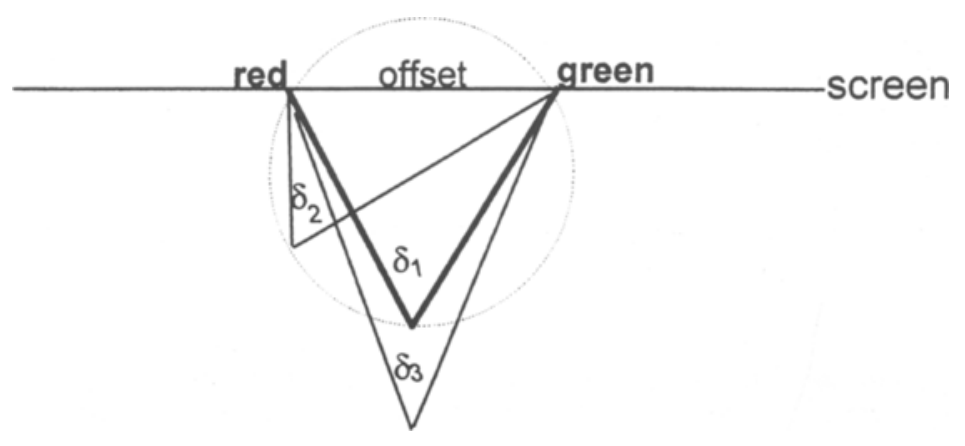

Figure 1. Changes in the disparity angle as the observer changes orientation or distance.

act with the geometric properties of the display in determining the apparent depth of the percept. The relative importance of the geometry and of the compensation processes is an empirical question. If perceptions follow geometric rules, depth should appear maximal when viewers are seated perpendicular to the screen (a position we refer to as the $0^{\circ}$ angle), and any change from this angle should lead to a decrease in the perceived depth. However, if compensation processes exist, the apparent depth should be less affected than expected from the geometrical predictions or may even remain unaffected by changing the orientation. It is important to emphasize that this compensation is irrelevant for the perception of real space, since changing the viewing orientation there yields different projections, allowing the exploration of the object's 3-D shape. It is only for artificial stimuli that the same projection is seen from different orientations and therefore subject to distortions, so that a specific experiment is needed to determine whether compensation for these distortions is possible.

The existence of compensatory processes was shown for the perception of 2-D pictures. A picture represents a specific scene only when seen from a single point of view. Nonetheless, we ordinarily view pictures from different points of view without experiencing distortions. It was suggested that mechanisms of perceptual compensation can enable viewers to counter the effects of projective transformations on depicted space (Kubovy, 1986; Pirenne, 1970). This claim was restricted by Goldstein (1987), who distinguished between two attributes of pictures. One of them, the spatial layout of objects in the pictures, remains relatively constant despite changes in the viewing angle. Another attribute, the orientation of objects relative to the observer, changes with viewing orientation, causing the differential rotation effect (Goldstein, 1987). It is this effect that causes the eyes of a portrait to "follow" a viewer who moves around a gallery.

These findings show that perception is not determined by constraints in the optical array only. The arrays for all views, except the one corresponding to the original station point, specify a distorted scene, and yet a key property of the picture remains invariant. Similar compensation processes may also exist in stereoscopic vision.
Such putative processes should be studied separately for the two attributes of a picture--the spatial layout and the orientation of objects relative to the observer.

The situation is very different with regard to incorrect viewing distances. The compensation to the correct orientation can be based on the assumption that the displays should be seen from a right angle, but no such intrinsic clue exists for the correct distance. Any viewing distance may appear equally legitimate, yet each will convey a different apparent depth. It is still unclear whether any distance can be accepted as correct, or whether the perceptual system assumes a default distance and compensates accordingly.

We noted that the geometry of stereopsis allows quantitative predictions of the magnitude of depth that should be perceived from different viewing distances. In the case of increasing distance, the angular difference between the two projections $(\delta)$ will decrease linearly as a function of the viewing distance. However, for a given disparity, depth is a nonlinear function of the distance (Equation 1). Therefore, changing the viewing distance will lead to a decrease in the disparity and an increase in the depth of the virtual object (the exact relations are given in the Appendix). If the visual system assumes and compensates for a specific "correct" distance, depth should remain unaffected by changing the viewing distance, or change less than expected from the geometrical predictions. This assumed distance cannot be predicted a priori.

The literature concerning binocular distance perception (and depth constancy) is large and diverse. Studies by Ellis and his collaborators and others suggest that observers tend to use an inappropriate value for their distance from the screen, when reconstructing spatial layout from perspective and stereoscopic information (Ellis, Tharp, Grunwald, \& Smith, 1991; Grunwald, Ellis, \& Smith, 1988; Hagen, 1993; McGreevy \& Ellis, 1986). Similarly, the work of Wallach et al. (1979) indicates that people do not estimate viewing distance correctly if the only available distance cues are convergence and accommodation. Some studies suggest that the distance to near targets is overestimated, the distance to far targets is underestimated, and for intermediate distances, percep- 
tion is close to veridical (Foley, 1980; Gogel, 1977; Johnston, 1991; Tittle et al., 1995; Todd et al., 1995). However, different studies used different distances and definitions of near and far. An explanation for this pattern of results could be that viewers assume that the correct distance is intermediate between the shorter and the larger distances that are used in the experiment, which would imply that the distance assessment depends on the context.

Our study attempts to determine the perceived depth of stereoscopic stimuli viewed from various orientations and distances. The experimental task required the estimation of stereoscopically presented angles. This task enabled us to study the perception of a spatial configuration without directly requiring the participants to evaluate the depth of individual points. Further, distortions of the configurations that result from changes in the viewing distance and orientation can be accurately predicted (Leiser et al., 1995). A nonlinear regression analysis of the participants' estimations can be used to reconstruct the viewing point that corresponds to the responses and to assess the degree of compensation manifested in the experiment.

\section{METHOD}

\section{Participants}

Six engineering students served as payed participants in the experiment. They had normal or corrected vision and successfully passed a commercial test of stereo acuity in which they identified raised circles in artificial stereoscopic stimuli, viewed through polarizing glasses. The circles' disparity increased systematically relative to their background, when seen from a distance of $40 \mathrm{~cm}$, from 800 to $40 \mathrm{~min}$ of arc. Only participants who were able to identify the target with a disparity of $50 \mathrm{~min}$ of arc or less participated in the experiment.

\section{Apparatus and Displays}

The stimuli were displayed on a PC clone with a color VGA screen. Responses were given by adjusting the angle of a large commercial caliper, formed by two $8.5-\mathrm{cm}$-long pivoted legs that could be opened to angles between $0^{\circ}$ and $180^{\circ}$. The stimuli were viewed through a set of commercial red/green stereoscopic viewing spectacles. A forehead restraint, mounted on a rod of adjustable length, was attached to the top of the monitor by a pivot. The forehead rest could be adjusted in any required direction. The stereoscopic stimuli were anaglyphs showing three points. One point (the apex) was located on the screen plane, and two other points (the leg endpoints) were symmetrically positioned on both sides and appeared to float in space (Figure 2a). In order to facilitate the participants' task, these two points were "in front" of the screen-that is, had crossed disparity (Patterson et al., 1992). The distance of the virtual points from the screen varied between stimuli, but all lay on two horizontal lines, perpendicular to the screen, ensuring that the 2-D location of a point provided no information about its 3-D depth (Figure 2b). The distance of the two lines from the central point was $2.5 \mathrm{~cm}$. The distances between the red and green projections of the floating points were computed to generate the correct disparity when seen from a distance of $60 \mathrm{~cm}$, directly in front of the screen.

\section{Tasks}

The participants were requested to perform two tasks. For $a b$ solute angle estimation, they held the caliper vertically in the fronto-parallel plane and opened it until its angle corresponded to the perceived angle. For relative orientation, the caliper was held

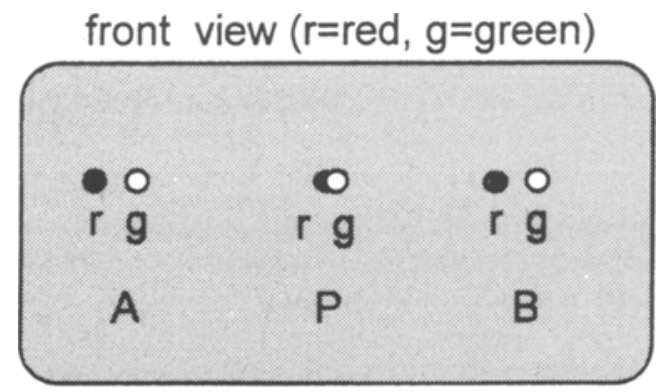

(a)

\section{top view}

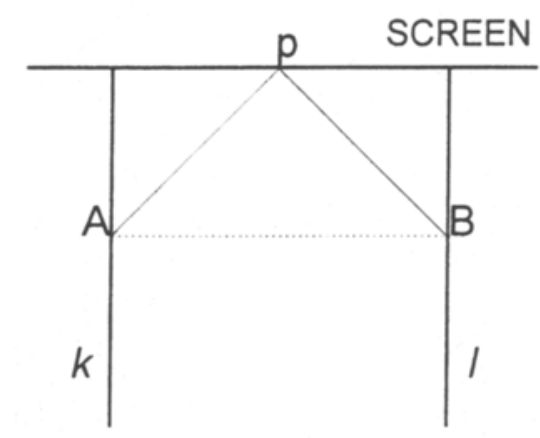

(b)

Figure 2. The experimental display: (a) front view of the stimulus presentation; (b) the geometry of the possible positions for the endpoints as seen from above. The apex was fixed, and the two endpoints appeared symmetrically on lines $\boldsymbol{k}$ and $\boldsymbol{l}$.

horizontally and participants were instructed to open its two legs until they were parallel to the legs of the perceived angle (as far as they could judge). The caliper was fully visible to the participant at all times. The stimuli remained on the screen until the participant was satisfied with the answer.

\section{Procedure and Design}

The experiments took place in a sparingly lit room, with the screen fully visible to the participants, who had therefore full cues to the orientation of the screen and distance to the display. In addition, the participant could also see the forehead restraint, which, as noted, was mounted on a straight telescopic metal rod fixed to the top of the viewing screen, and this provided the participants with usable information about their orientation and distance.

Participants saw the displays from one of four orientations $\left(0^{\circ}\right.$, $35^{\circ}, 55^{\circ}$, or $70^{\circ}$, with the position perpendicular to the screen considered as $\left.0^{\circ}\right)$ and at one of three distances $(40,60$, or $80 \mathrm{~cm})$. Each combination of orientation and distance formed a block. Every block began with five practice trials, followed by three repetitions of seven displayed angles $\left(38^{\circ}, 60^{\circ}, 79^{\circ}, 94^{\circ}, 114^{\circ}, 140^{\circ}\right.$, and $\left.171^{\circ}\right)$. The order of presentation of the different angles was randomized within each block. The 12 combinations of orientation $\times$ distance were randomly assigned to three sessions of four blocks each. Because there were two tasks (absolute angle estimation and relative orientation), each participant attended six sessions in all. Three participants performed absolute angle estimation in the first three sessions and relative orientation in the remaining three, and the other participants performed the tasks in the reverse order. 


\section{RESULTS}

For each participant and block (i.e., orientation $\times$ distance combination), we computed the mean estimate across the three repetitions of each angle. This was done separately for the two tasks.

The data were analyzed using a four-way analysis of variance (ANOVA) with repeated measures. The dependent variable was the estimated angle and the independent variables were the task, the viewing distance, the viewing orientation, and the presented angles. Full compensation for distance or orientation should express itself in nonsignificant main effects and interactions of the variables. The ANOVA revealed significant main effects of the viewing orientation $[F(3,15)=16.58, p<.001]$, the presented angles $[F(6,30)=69.34, p<.001]$, and a significant interaction between the viewing orientation and the presented angle $[F(18,90)=3.017, p<.001]$, shown in Figure 3. All effects are consistent in their direction with the predictions of the mathematical function, according to which the estimated angle should increase as a function of the viewing orientation and the size of the presented angle, and these two variables should interact. No main effects and interactions were found for the task or the viewing distance. Means and standard deviations for the different conditions across tasks are reported in Table 1.
Although the significant main effects and interactions of the orientation indicate that there is no full compensation, the extent of this lack of compensation is not clear. In order to evaluate this, one has to determine the viewing position that corresponds to the estimated angles. To this end we performed nonlinear regressions designed to reconstruct the distances and the orientations that would yield estimates that resemble participants' responses.

The mathematical function specified in the Appendix was used as the nonlinear function, leading to the regression equation shown in the Appendix. Assuming that the compensations are best expressed as the ratio of the best-fit distance and orientation to their actual values, we estimated two parameters: $b_{d}$ (the proportion of the best-fit distance to the actual viewing distance) and $b_{o}$ (the proportion of the best-fit orientation to the actual viewing orientation). The interocular distance was taken to be $6.3 \mathrm{~cm}$ (Ono \& Comerford, 1977). We did not measure individual values to fit the function because all participants were young male adults, and the variability is known to be small. The dependent variable was the estimated angle, and the independent variables were the viewing distance and the orientation. The regression analyses were conducted across tasks.

In this regression, $S S_{\text {reg }}=2,815.85$ and $S S_{\text {res }}=1,095.37$, and the ratio of $S S_{\text {reg }}$ and $S S_{\text {res }}$ was 0.72 , indicating a

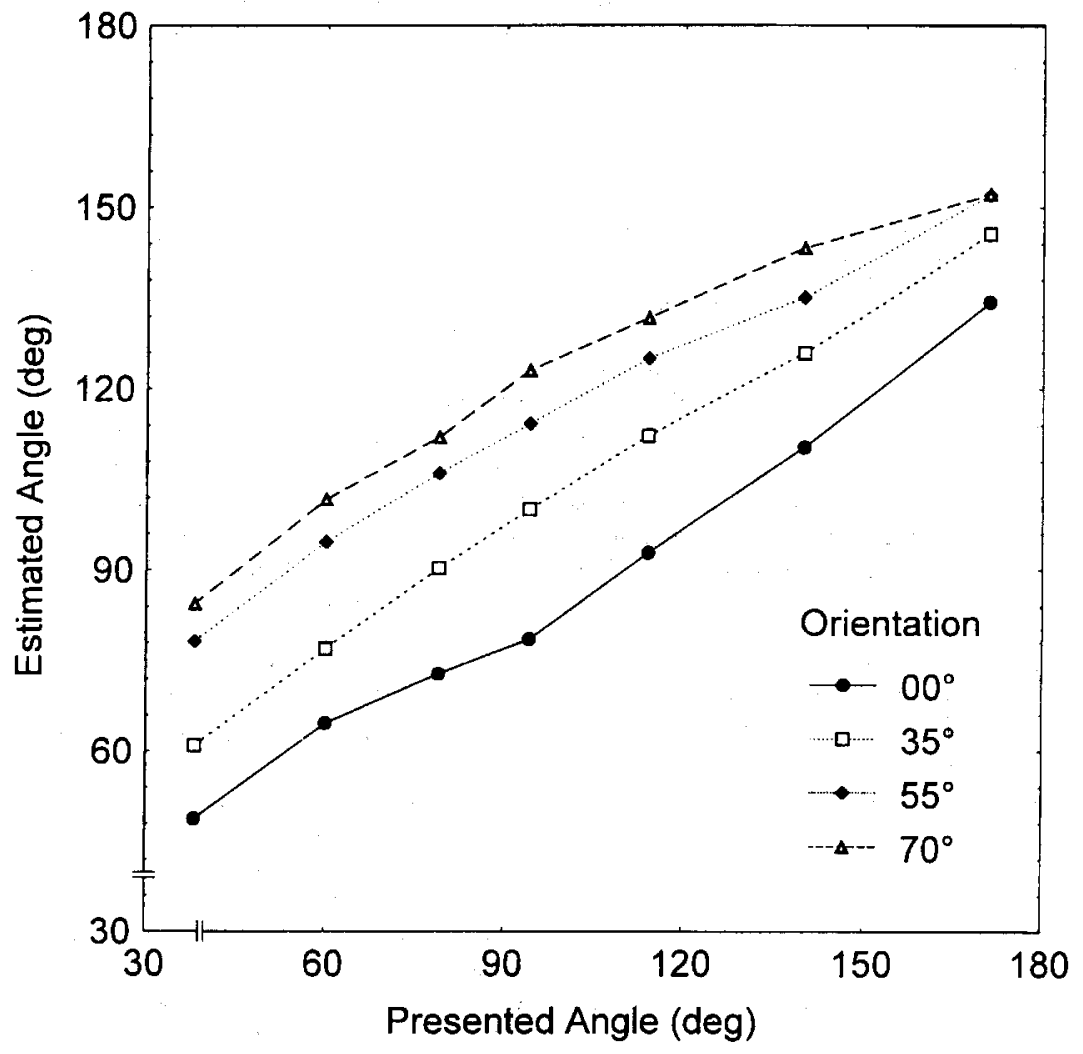

Figure 3. Mean estimated angle as a function of the presented angle for the four viewing orientations across tasks. 
Table 1

Means and Standard Deviations for the Experimental Conditions

\begin{tabular}{|c|c|c|c|c|c|c|c|c|c|}
\hline \multirow[b]{3}{*}{ Distance } & \multirow[b]{3}{*}{ Angle } & \multicolumn{8}{|c|}{ Orientation } \\
\hline & & \multicolumn{2}{|c|}{$0^{\circ}$} & \multicolumn{2}{|c|}{$35^{\circ}$} & \multicolumn{2}{|c|}{$55^{\circ}$} & \multicolumn{2}{|c|}{$70^{\circ}$} \\
\hline & & $M$ & $S D$ & $M$ & $S D$ & $M$ & $S D$ & $M$ & $S D$ \\
\hline \multirow[t]{7}{*}{$40 \mathrm{~cm}$} & $38^{\circ}$ & 48.7 & 20.6 & 59.4 & 26.1 & 72.0 & 41.0 & 85.7 & $\overline{37.6}$ \\
\hline & $60^{\circ}$ & 65.3 & 25.8 & 75.9 & 32.7 & 91.0 & 46.3 & 105.7 & 38.8 \\
\hline & $79^{\circ}$ & 72.7 & 21.3 & 91.1 & 32.8 & 99.6 & 46.6 & 120.4 & 33.9 \\
\hline & $94^{\circ}$ & 80.8 & 23.8 & 97.1 & 32.9 & 109.0 & 47.1 & 132.4 & 28.8 \\
\hline & $114^{\circ}$ & 98.1 & 20.1 & 111.8 & 33.0 & 118.4 & 43.0 & 135.9 & 23.2 \\
\hline & $140^{\circ}$ & 116.1 & 16.1 & 126.6 & 28.8 & 126.3 & 40.2 & 143.9 & 22.5 \\
\hline & $171^{\circ}$ & 139.2 & 16.9 & 144.7 & 20.5 & 147.4 & 25.8 & 149.5 & 15.8 \\
\hline \multirow[t]{7}{*}{$60 \mathrm{~cm}$} & $38^{\circ}$ & 47.1 & 21.0 & 58.9 & 26.3 & 74.6 & 28.6 & 82.7 & 36.1 \\
\hline & $60^{\circ}$ & 59.6 & 23.5 & 75.7 & 30.6 & 90.5 & 21.3 & 95.4 & 34.1 \\
\hline & $79^{\circ}$ & 68.8 & 21.8 & 82.9 & 29.9 & 104.0 & 21.4 & 108.4 & 31.5 \\
\hline & $94^{\circ}$ & 77.3 & 22.9 & 96.0 & 30.5 & 114.7 & 16.5 & 121.3 & 25.4 \\
\hline & $114^{\circ}$ & 92.2 & 20.0 & 105.4 & 28.3 & 125.3 & 14.7 & 131.2 & 20.0 \\
\hline & $140^{\circ}$ & 105.9 & 16.0 & 118.2 & 24.7 & 137.3 & 11.9 & 141.7 & 18.4 \\
\hline & $171^{\circ}$ & 129.4 & 16.5 & 140.3 & 18.6 & 152.8 & 10.3 & 154.4 & 12.9 \\
\hline \multirow[t]{7}{*}{$80 \mathrm{~cm}$} & $38^{\circ}$ & 50.4 & 28.1 & 64.4 & 33.6 & 88.0 & 23.2 & 84.8 & 31.2 \\
\hline & $60^{\circ}$ & 69.0 & 32.5 & 79.4 & 26.9 & 102.5 & 32.3 & 104.1 & 34.2 \\
\hline & $79^{\circ}$ & 77.1 & 32.9 & 97.0 & 33.1 & 114.6 & 25.1 & 107.4 & 34.1 \\
\hline & $94^{\circ}$ & 77.5 & 26.2 & 106.8 & 35.1 & 118.9 & 28.1 & 115.5 & 30.8 \\
\hline & $114^{\circ}$ & 88.2 & 26.1 & 119.6 & 30.2 & 131.2 & 20.0 & 128.1 & 25.8 \\
\hline & $140^{\circ}$ & 109.0 & 21.0 & 132.9 & 21.8 & 141.8 & 14.5 & 144.3 & 14.9 \\
\hline & $171^{\circ}$ & 134.5 & 17.1 & 151.9 & 15.9 & 156.1 & 12.5 & 152.8 & 13.9 \\
\hline
\end{tabular}

moderate fit of the regression with these parameters. For the viewing distance, $b_{d}=2.08$, which means that participants overestimated the distances; for the orientation, $b_{o}=0.4$, which is less than unity and larger than zero, suggesting that participants compensate partially for the incorrect viewing orientation.

The results are presented graphically in Figure 4, which shows a $3 \times 3$ matrix of panels. The top panel presents the geometric predictions for each presented angle from each viewing orientation, the middle panel presents the mean estimates across tasks for each presented angle from each viewing orientation, and the bottom panel presents the geometric predictions for each presented angle from each viewing orientation according to the set of parameters that was found in the regression analysis. Each column corresponds to a different viewing distance. The geometric predictions were computed from the mathematical function in the Appendix, taking into account the orientation, the distance, and the presented angle. The graphs in the nine panels have the same layout. The $x$-axis shows the presented angle in degrees, and the $y$-axis is the estimated (or predicted) angle in degrees. The different line patterns correspond to viewing orientations, as described in the legend.

The comparison of the geometric prediction to the data in Figure 4 indicates that participants' judgments were less affected by the viewing orientation than was predicted by the mathematical function. This finding is in accordance with the partial compensation that is implied by the value of the orientation's parameter. However, the comparison of the data to the predictions that were computed with the regression parameters revealed differences. Whereas the mathematical function predicts very similar estimates for all orientations, the actual estimates change as a function of the orientation. It seems that the mathematical function with a single set of parameters for all viewing orientations is not adequate as a descriptive model. In order to better understand the meaning of these parameters, we analyzed the results separately for each distance and for each orientation.

The interpretation of the distance parameter could be misleading since $b_{d}=1$ may not necessarily imply that there was no compensation. For instance, participants could underestimate distances larger than $60 \mathrm{~cm}$ and overestimate distances smaller than $60 \mathrm{~cm}$, a pattern that was found in previous experiments (Foley, 1980; Gogel, 1977; Johnston, 1991). We therefore also estimated $b_{d}$ separately for every distance. The results are presented in Table 2 .

The results show that all $b_{d}$ values are larger than unity. Participants always overestimate their distance from the screen and do not compensate to the correct distance of $60 \mathrm{~cm}$ (this would have yielded coefficients larger than 1 for the distance of $40 \mathrm{~cm}$, of 1 for $60 \mathrm{~cm}$, and smaller than 1 for $80 \mathrm{~cm}$ ). Indeed, they do not compensate for the increased distance at all, as would have been indicated by decreasing coefficients for larger distances. The columns in Table 2 present the reconstructed distances that correspond to the $b_{d}$ coefficients obtained by multiplying the actual seating distance by the $b_{d}$ coefficients. Participants consistently overestimated their distance from the screen by a factor of 2 .

We also computed separate regressions for each nonzero orientation. The zero orientation was not included because in this case the parameters are multiplied by zero, making it impossible to distinguish among param- 
Geometric prediction

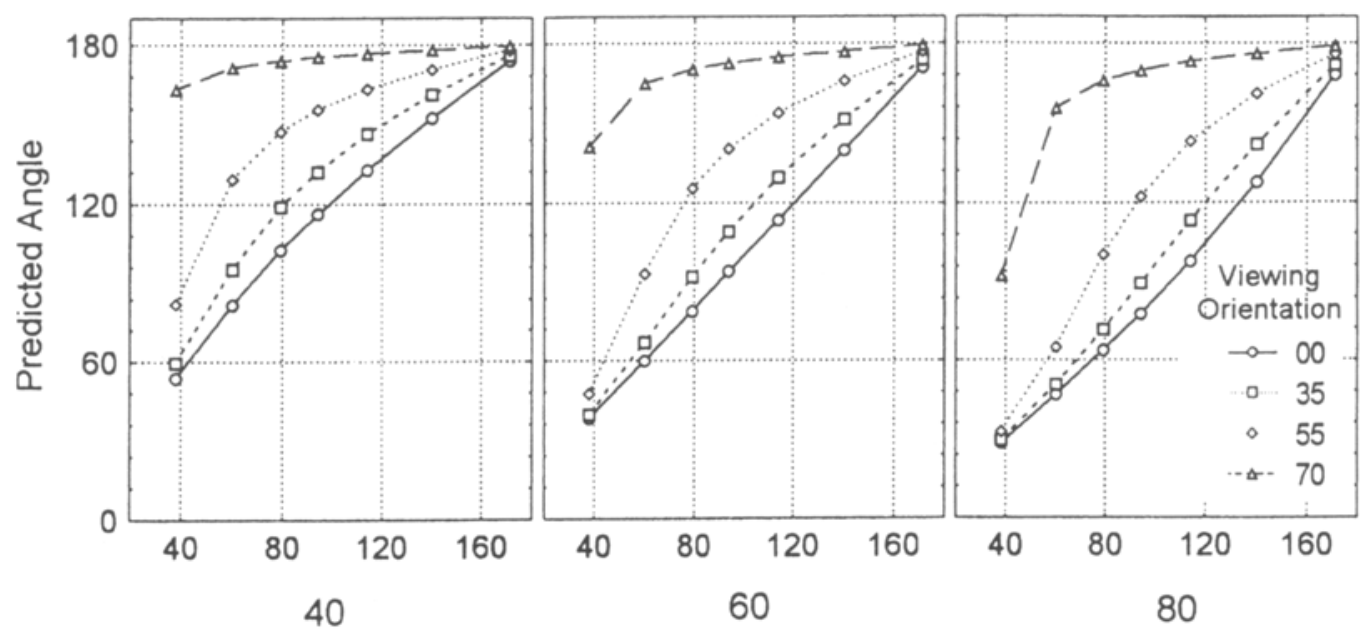

Judgments across tasks
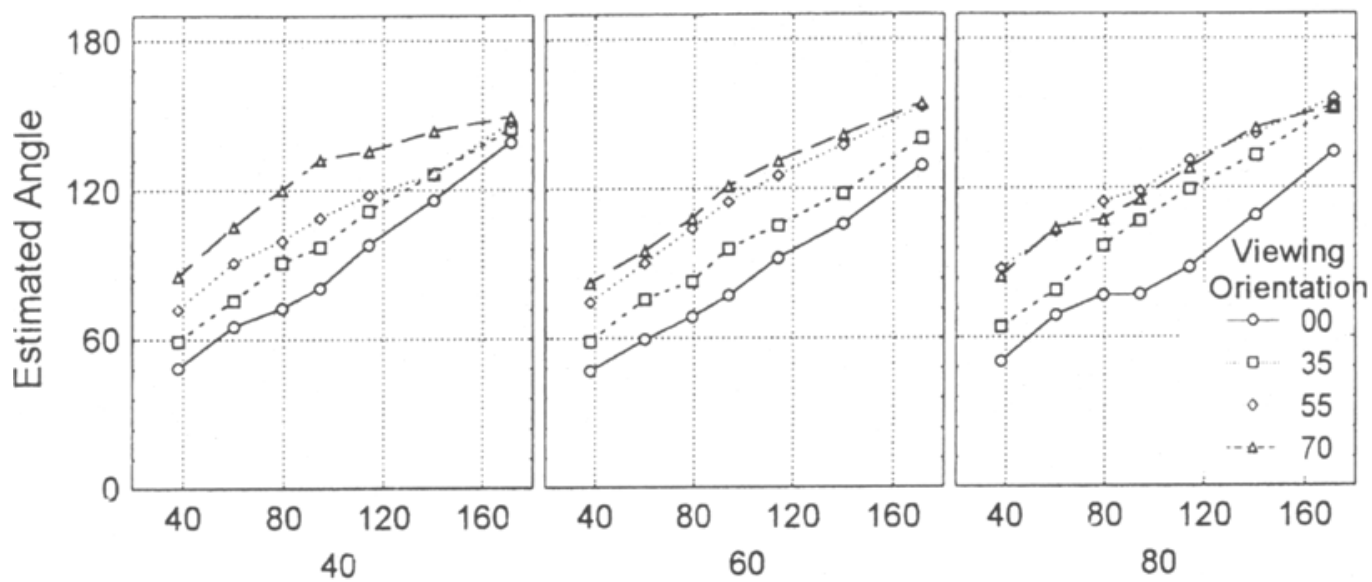

\section{Prediction according to regression results}

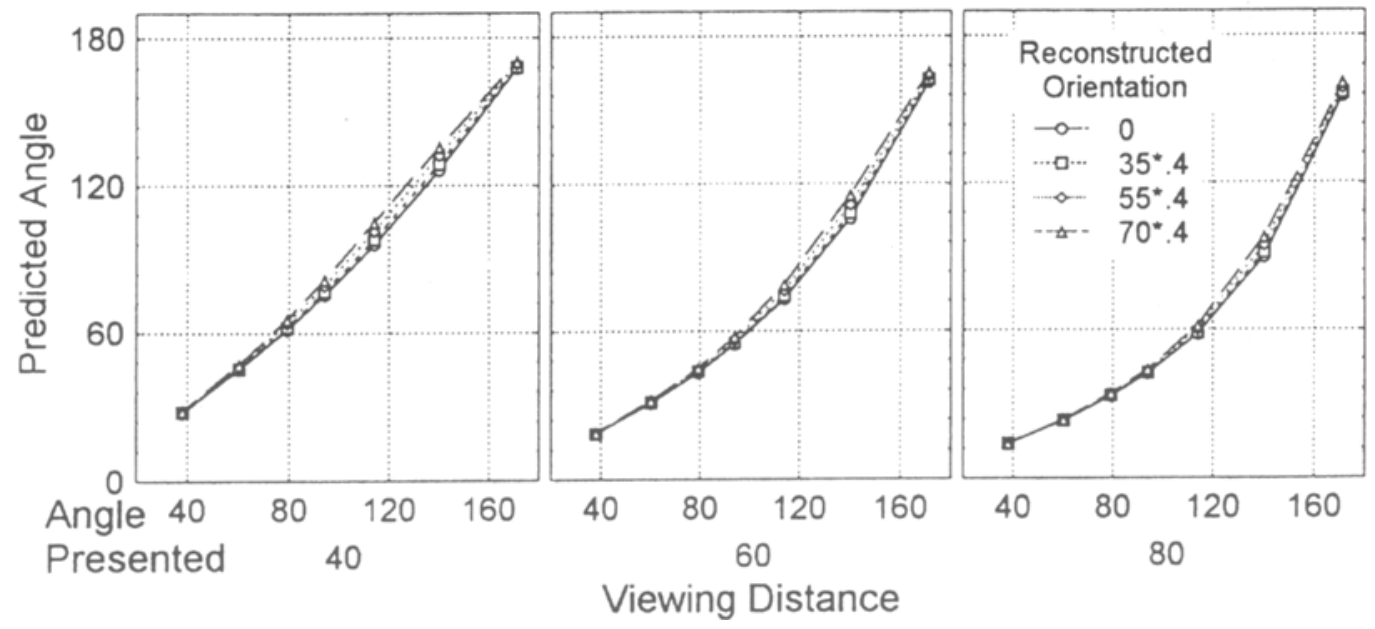

Figure 4. Comparison between geometric predictions (top panel), mean estimates across tasks (middle panel), and geometric predictions according to the set of parameters that was found in the regression analysis (bottom panel) for the different viewing distances and orientations. 
Table 2

Results of Separate Nonlinear Regressions for the Viewing Distances: Nonlinear Regression Coefficients for Each Distance $\left(b_{o}=2\right)$

\begin{tabular}{ccccc}
\hline Distance & $b_{d}$ & $\begin{array}{c}\text { Reconstructed } \\
\text { Distance }\end{array}$ & $\mathrm{SS}_{\text {reg }}$ & SS $_{\text {res }}$ \\
\hline 40 & 2.12 & 84.8 & 938.25 & 383.84 \\
60 & 2.24 & 134.4 & 866.00 & 363.11 \\
80 & 1.94 & 155.2 & $1,013.01$ & 346.99 \\
\hline
\end{tabular}

Note $-\mathrm{SS}_{\text {reg }}$, sum squares of the regression; $\mathrm{SS}_{\text {res }}$, sum squares of the residuals.

Table 3

Results of Separate Nonlinear Regressions for the Viewing Orientations: Nonlinear Regression Coefficients for Each Viewing Orientation $\left(b_{d}=0.4\right)$

\begin{tabular}{cccrr}
\hline Orientation & $b_{o}$ & $\begin{array}{c}\text { Reconstructed } \\
\text { Orientation }\end{array}$ & \multicolumn{1}{c}{$\mathrm{SS}_{\text {reg }}$} & \multicolumn{1}{c}{$\mathrm{SS}_{\text {res }}$} \\
\hline $35^{\circ}$ & 0.85 & $30^{\circ}$ & 908.40 & 114.70 \\
$55^{\circ}$ & 0.37 & $20^{\circ}$ & $1,037.15$ & 83.76 \\
$70^{\circ}$ & 0.25 & $17^{\circ}$ & $1,148.67$ & 72.09 \\
\hline
\end{tabular}

Note- $-\mathrm{SS}_{\mathrm{reg}}$, sum squares of the regression; $\mathrm{SS}_{\text {res }}$, sum squares of the residuals.

eters. The values $b_{o}$ of decrease as a function of the orientation. This decrease can serve as evidence for a compensation process. The results are presented in Table 3 . Reconstructed orientations that correspond to the $b_{o}$ coefficients, computed by multiplying the actual orientation by the $b_{o}$ coefficients, ranged from $17^{\circ}$ to $30^{\circ}$. This finding reinforces our belief that a compensation process took place. The ratio of the sums of the regression to the total sum of squares was about 0.90 for each orientation, and it indicates that the fit of the regression with these parameters is extremely good. However, this model has many parameters, and thus one cannot rule out the possibility that other models that do not assume compensation can account for these data equally well.

\section{DISCUSSION}

Stereoscopic displays (like most other displays) are designed under the assumption that people are seated perpendicular to the center of the screen. The geometric analysis of artificial stereoscopic stimuli leads to the prediction of systematic distortions of the image that result from changing the viewing distance and the orientation from which the display is seen.

For 2-D pictures, Goldstein (1987) distinguished between tasks that require the determining of the spatial layout and tasks that require indicating the perceived orientation of elements in the picture. The former task was performed accurately, regardless of the viewing angle, showing the use of compensatory mechanisms. Perceived orientation, however, was systematically distorted as a function of the viewing angle. In order to determine whether a similar difference exists in tasks involving stereoscopic stimuli, we asked participants to adjust a caliper so that its sides were parallel to the perceived angle (a task that we considered equivalent to indicating the perceived orientation of elements) and to adjust a vertically held caliper until the angle corresponded to the displayed angle (a task we considered equivalent to reproducing the spatial layout in the display). There is no evidence for any difference in the performance of these tasks with stereoscopic displays, where, it seems, spatial layout and orientation to the viewer cannot be dissociated.

Our study aimed to examine the extent to which people's percepts correspond to the mathematical predictions. When participants' responses were compared with the mathematical function with respect to viewing distance and viewing orientation, different patterns of results emerged. There was no compensation for the viewing distance, and partial compensation for the viewing orientation. One should keep in mind that the stimuli held no clues for the correct viewing distance. This is not a result of the experimental setup, which afforded full view of the screen and its surroundings, but a logical point: There is no basis for deciding, upon seeing a 3-D angle stereoptically, whether the viewing distance is appropriate. It would have been possible for participants to postulate a "correct" distance - for instance, the distance from which people usually view a monitor. We found that people overestimate distances, and they do so to an increasing extent when the seating distance is increased. This finding is in line with other research that yielded similar results (Johnston, 1991; Tittle et al., 1995; Todd et al., 1995). It seems therefore that participants had no prior assumption as to the correct viewing distance, and that they assumed that the correct distance was partly a function of where they were seated.

Although the correct viewing distance cannot be determined, the correct viewing orientation is naturally perpendicular to the screen. It is an empirical question whether participants compensate to this orientation. The results demonstrate that participants did not fully compensate for the incorrect viewing orientation. Yet, comparison of participants' estimates to the geometric predictions revealed differences. These differences suggested that some compensation may exist. A quantitative analysis of these results with a nonlinear regression on the geometric function provides good fit, as long as we assumed different parameters for the different orientations. The different parameters imply that the proportion of compensation increases as the viewing orientation moves away from the perpendicular position. Although our findings are less extreme than previous results on the perception of nonstereoptic pictures viewed from a wrong station point, the appearance of compensationlike processes is in line with them (e.g., Kubovy, 1986; Rosinski, Mulholland, Degelman, \& Farber, 1980). However, compensation processes are but one possible explanation for the results, and other models that do not assume compensation may yet describe the pattern of results equally well. 
The analysis of the interaction between the two sources of distortion (distance and orientation) suggests that the two are independent. Compensation to the correct orientation is partially possible and is performed by the participants, whereas compensation to the distance suffers from uncertainty as to the correct viewing distance.

\section{REFERENCES}

CoRmack, R. H. (1984). Stereoscopic depth perception at far viewing distances. Perception \& Psychophysics, 35, 423-428.

Ellis, S. R., TharP, G. K., Grunwald, A. J., \& Smith, S. (1991). Exocentric judgments in real environments and stereoscopic displays. In Proceedings of the Human Factors Society 35 th Annual Meeting (pp. 1442-1446). Santa Monica, CA: Human Factors Society.

Foley, J. M. (1980). Binocular distance perception. Psychological Review, 87, 411-434.

Fox, R., Cormack, L., \& Norman, J. F. (1987). The effect of vertical disparity on depth scaling. Investigative Ophthalmology \& Visual Science, 28 (Suppl.), 293.

GoGEL, W. C. (1977). An indirect measure of perceived distance from oculomotor cues. Perception \& Psychophysics, 21, 3-11.

GolDSteIN, E. B. (1987). Spatial layout, orientation relative to the observer and perceived projection in pictures viewed at an angle. Journal of Experimental Psychology. Human Perception \& Performance, $13,256-266$

Grunwald, A. J., Ellis, S. R., \& SMith, S. (1988). A mathematical model for spatial orientation from pictorial perspective displays. IEEE Transactions on Systems, Man, \& Cybernetics, 18, 425-437.

HAGEN, M. A. (1993). Where's the camera? Ecological Psychology, 5, 65-84.

JoHnSTON, E. B. (1991). Systematic distortion of shape from stereopsis. Vision Research, 31, 1351-1360.

Kubovy, M. (1986). The psychology of perspective and Renaissance art. London: Cambridge University Press.

Leiser, D., Bereby, Y., \& Melkman, A. (1995). Stereoscopic projection and seating arrangements. Ergonomics, 38, 1231-1238.

McGreevy, M. W., \& Ellis, S. R. (1986). The effect of perspective geometry on judged direction in spatial information instruments. Human Factors, 28, 439-456.

Ono, H., \& Comerford, J. (1977). Stereoscopic depth constancy. In W. Epstein (Ed.), Stability and constancy in visual perception (pp. 91-128). New York: Wiley.

Patterson, R., Moe, L., \& Hewitt, T. (1992). Factors that affect depth perception in stereoscopic displays. Human Factors, 34, 655-667.

PirenNe, M. H. (1970). Optics, painting and photography. Cambridge: Cambridge University Press.

RITTER, M. (1977). Effect of disparity and viewing distance on perceived depth. Perception \& Psychophysics, 22, 400-407.

Ritter, M. (1979). Perception of depth: Processing of simple positional disparity as a function of viewing distance. Perception \& Psychophysics, 25, 209-214.

Rosinski, R. R., Mulholland, T., Degelman, D., \& Farber, J. (1980). Picture perception: An analysis of visual compensation. Perception \& Psychophysics, 28, 521-526.

Tittle, J. S., Todd, J. T., Perotti, V. J., \& Norman, J. F. (1995). Systematic distortion of perceived three-dimensional structure from motion and binocular stereopsis. Journal of Experimental Psychology: Human Perception \& Performance, 21, 663-678.

Todd, J. T., TitTle, J. S., \& Norman, J. F. (1995). Distortions of threedimensional space in the perceptual analysis of motion and stereo. Perception, 24, 75-86.

Wallach, H., Gillam, B., \& Cardillo, L. (1979). Some consequences of stereoscopic depth constancy. Perception \& Psychophysics, 26, 235-240.

\section{APPENDIX}

Following is an explanation of the mathematical computations that were used in the paper. First we show how displacements in orientation and distance affect the perceived location of a point. These computations have already been presented elsewhere (Leiser, Bereby, \& Melkman, 1995), but we repeat them for the sake of completeness. They provide the basis for the computation of the changes of an angle that is defined by three points. They are also used in a nonlinear regression function to assess the distance and orientation that correspond to participants' responses.

As is usual, we assume a left-handed coordinate system, with the screen as $x, y$ plane for the correctly seated observer. At the correct seating the observer's eyes are parallel to the screen and at a (perpendicular) distance of $d_{o}$ from the center of the screen. The intereye distance of the observer is $I$. The displaced seating is one where the observer is rotated by an angle $\alpha$ (counterclockwise positive, in the $x, z$ plane) with respect to the center of the screen and placed at a distance $d_{n}$. In what follows we develop the equation for the $(x, y)$ coordinate only.

A. The screen coordinates of a point $P=(x, z)$ (Figure A1, top panel). Denote the observer's right eye by $R=$ $\left(1 / 2 I,-d_{0}\right)$ and let $P_{R}=\left(X_{R}, 0\right)$ be the screen coordinates of $P$ as seen by $R$. From the similarity of triangles ARP and $\mathrm{BRP}_{R}$ follows

$$
\frac{x_{R}-\frac{1}{2} I}{x-\frac{1}{2} I}=\frac{d_{o}}{d_{o}+z},
$$

that is,

$$
x_{R}=\frac{d_{o} x+\frac{1}{2} l z}{d_{o}+z}
$$

Similarly,

$$
x_{L}=\frac{d_{o} x-\frac{1}{2} l z}{d_{o}+z} .
$$

B. $P^{\prime}$, the location of $P$ after rotation and perpendicular displacement (Figure A1, bottom panel). We can now calculate the coordinates of $P^{\prime}=\left(x^{\prime}, z^{\prime}\right)$ with respect to the rotated coordinate system (indicated by primes). With respect to this coordinate system, $P_{R}=\left(x_{R} \cos \alpha,-x_{R} \sin \alpha\right)$. Using the similarity of triangles $A^{\prime} R^{\prime} P^{\prime}$ and $C R^{\prime} P_{R}$ we obtain

$$
\frac{x^{\prime}-\frac{1}{2} I}{x_{R} \cos \alpha-\frac{1}{2} I}=\frac{d_{n}+z^{\prime}}{d_{n}-x_{R} \sin \bar{\alpha}}
$$

and similarly

$$
\frac{x^{\prime}+{ }_{2}^{1} I}{x_{L} \cos \alpha+\frac{1}{2} I}=\frac{d_{n}+z^{\prime}}{d_{n}-x_{L} \sin \alpha} .
$$

Solving these equations for $x^{\prime}$ and $z^{\prime}$ yields

$x^{\prime}=\frac{I}{D(\alpha)}\left(d_{n} \frac{x_{L}+x_{R}}{2} \cos \alpha+I \frac{x_{L}-x_{R}}{4} \sin \alpha-x_{L} x_{R} \cos \alpha \sin \alpha\right)$

and 


\section{APPENDIX (Continued)}

$$
z^{\prime}=\frac{I}{D(\alpha)}\left(-I d_{n} \frac{x_{L}+x_{R}}{2} \sin \alpha-I d_{n}^{2}\left(x_{L}-x_{R}\right) \cos \alpha+I x_{L} x_{R} \sin ^{2} \alpha\right)
$$

with

$$
D(\alpha)=I d_{n}+d_{n}\left(x_{L}-x_{R}\right) \cos \alpha-I \frac{x_{L}+x_{R}}{2} \sin ^{2} \alpha
$$

on the basis of the equations derived above.

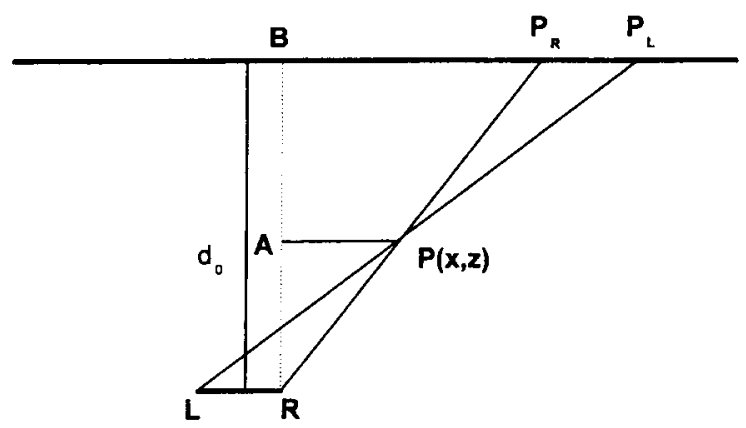

Before the position change

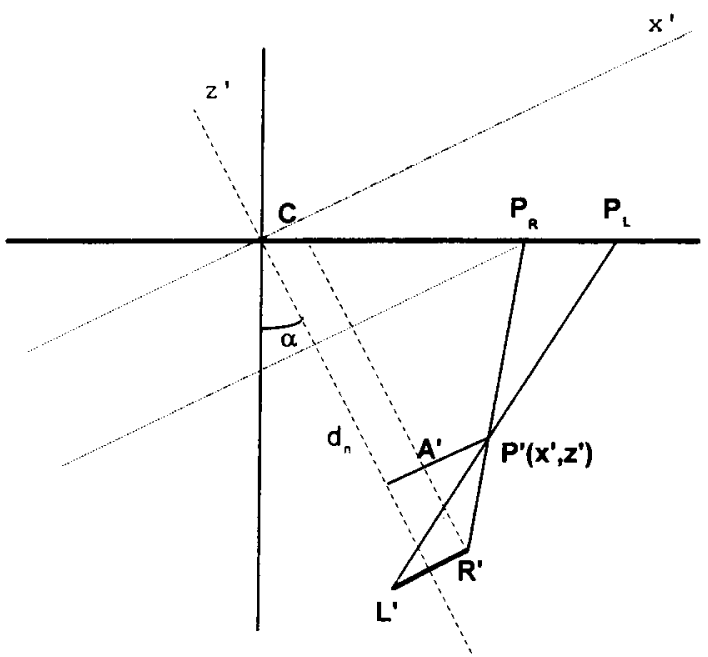

After the position change

Figure A1. Change of the apparent position of a point as a function of an observer's displacement.
C. Distortions of an angle after rotation and perpendicular displacement. Given three points that when viewed from the correct position have the coordinates $\left(x_{1}, z_{1}\right),(0,0)$, and $\left(x_{2}, z_{2}\right)$, these points define an angle $A$, which following the law of cosines is

$$
A=\operatorname{acos}\left(\frac{x_{1} x_{2}+z_{1} z_{2}}{\sqrt{\left[\left(x_{1}\right)^{2}+\left(z_{1}\right)^{2}\right]\left[\left(x_{2}\right)^{2}+\left(z_{2}\right)^{2}\right]}}\right) .
$$

Using Equations $\mathrm{A} 3$ and $\mathrm{A} 4$, it is possible to compute $A^{\prime}$ after the displacement accordingly.

D. The nonlinear regression. In order to reconstruct the position that corresponds to an observer's angle estimates, a nonlinear regression can be employed, using Equations A3 and A4 and the coefficients $b_{o}$ for the orientation and $b_{d}$ for the distance. The equations are then

$$
\begin{aligned}
x^{\prime}=\frac{I}{D(\alpha)}\left(b_{d} d_{n} \frac{x_{L}+x_{R}}{2} \cos \left(b_{o} \alpha\right)\right. \\
\\
\left.\quad+I \frac{x_{L}-x_{R}}{4} \sin \left(b_{o} \alpha\right)-x_{L} x_{R} \cos \left(b_{o} \alpha\right) \sin \left(b_{o} \alpha\right)\right)
\end{aligned}
$$

$$
\begin{aligned}
z^{\prime}=\frac{I}{D(\alpha)}\left(-I b_{d} d_{n} \frac{x_{L}+x_{R}}{2} \sin \left(b_{o} \alpha\right)\right. \\
\left.\quad-I b_{d}^{2} d_{n}^{2}\left(x_{L}-x_{R}\right) \cos \left(b_{o} \alpha\right)+I x_{L} x_{R} \sin ^{2}\left(b_{o} \alpha\right)\right)
\end{aligned}
$$

with

$$
D(\alpha)=I b_{d} d_{n}+b_{d} d_{n}\left(x_{L}-x_{R}\right) \cos \left(b_{o} \alpha\right)-I \frac{x_{L}+x_{R}}{2} \sin ^{2}\left(b_{o} \alpha\right) .
$$

Predictions of an observer's estimates $A_{e s t}$ can then be made using the function

$$
A_{e s t}=\operatorname{acos}\left(\frac{x_{1}^{\prime} x_{2}^{\prime}+z_{1}^{\prime} z_{2}^{\prime}}{\sqrt{\left[\left(x_{1}^{\prime}\right)^{2}+\left(z_{1}^{\prime}\right)^{2}\right]\left[\left(x_{2}^{\prime}\right)^{2}+\left(z_{2}^{\prime}\right)^{2}\right]}}\right) .
$$

(Manuscript received December 5, 1994; revision accepted for publication July 27, 1998.) 